\title{
EL MODELO HALL-TONNA COMO HERRAMIENTA DE DESARROLLO DE LAS CUATRO «C'S» EN LOS COLEGIOS DE LA COMPAÑÍA DE JESÚS
}

\author{
JOSÉ ANTONIO ORTÍ MARTÍNEZ ${ }^{1}$
}

Fecha de recepción: octubre 2018

Fecha de aceptación y versión definitiva: octubre 2019

RESUMEN: El presente artículo muestra la vinculación existente entre el modelo de análisis de valores Hall-Tonna y el paradigma pedagógico ignaciano. Se describe cómo el modelo puede suponer una herramienta de aprendizaje clave para el desarrollo de las cuatro cualidades objetivo de la educación jesuita: formar personas conscientes, competentes, compasivas y comprometidas, ayudando a la transformación de los colegios de la Compañía de Jesús en organizaciones que aprenden.

PALABRAS CLAVE: valores; aprendizaje; educación; organización que aprende.

\section{The Hall-Tonna Model as a tool for the development of the four "C's" in the schools of the Company of Jesus}

ABSTRACT: This article shows the link between the Hall-Tonna values analysis model and the Ignatian pedagogical paradigm. It describes how the model can be a key learning tool for the development of the four qualities that are the objective of Jesuit education: to form conscious, competent, compassionate and committed people. This supports the transformation of the schools of the Society of Jesus into organizations that learn.

KEY WORDS: values; learning; education; learning organizations.

1 Docente en la Universidad Católica San Antonio de Murcia. Investigador en la Cátedra de Liderazgo e Internacionalización de la Universidad Pontificia Comillas. Correo electrónico: jorti@ucam.edu. 


\section{INTRODUCCIÓN}

El modelo Hall-Tonna tiene como objeto principal el análisis de los valores en organizaciones a través de un proceso de transformación y conversión del conocimiento que "permite identificar y gestionar el conflicto posibilitando procesos de aprendizaje en base a valores» (Elexpuru y Bunes, 1994, p. 7). El análisis parte de los valores personales hasta llegar a los de la organización, puesto que una vez que los individuos aclaran sus prioridades de valor y visiones personales están en disposición de interactuar abiertamente en un proceso de definir valores compartidos y construir un consenso sobre los valores fundamentales de la institución.

Los antecedentes del modelo Hall-Tonna en España, vinculados al mundo educativo, tienen su origen en la Universidad de Deusto. Algunos de los profesores adheridos a dicha Universidad publicaron un libro dirigido por Elexpuru y Medrano (Elexpuru y Medrano, 2001) que tenía como objetivo investigar sobre la cultura de los centros educativos y la educación en valores. Otros libros o artículos como Los valores de la LOGSE: análisis según la metodología de Hall-Tonna (Bunes et al., 1993) se centraron en el estudio de los valores implícitos y explícitos contenidos en la Ley Orgánica 1/1990, de 3 de octubre, de Ordenación General del Sistema Educativo (BOE núm. 238, jueves 4 octubre 1990). Posteriormente, en la publicación de 2013 de Elexpuru, Villardón y Yániz (Elexpuru, Villardón y Yániz, 2013), los autores identificaron los valores de los alumnos, profesores y centros educativos como punto de partida para realizar una propuesta de desarrollo de los valores institucionales. Por otro lado, en el libro de 2003 de Fonseca (Fonseca, 2003) se llevó a cabo un análisis de los valores de los docentes relacionando estos con los principios de la teoría del desarrollo moral de Kohlberg (Kohlberg, 1984).

El modelo Hall-Tonna adopta los apellidos de sus dos creadores: Brian P. Hall y Benjamin Tonna. Hall ha sido catedrático de consejo psicológico y educación en la Universidad de Santa Clara (California), propiedad de la Compañía de Jesús, la cual forma parte de la Asociación de Universidades Jesuitas (IAJU) en la que se integran las 28 universidades que la Compañía de Jesús dirige en Estados Unidos. Hall fundó y presidió Values Technology, empresa que desde la década de los 70 del siglo XX tiene como misión ayudar al desarrollo personal y la transformación de equipos y organizaciones a través de una mejor comprensión de sus valores y su liderazgo. Hall ha estado involucrado durante más de 40 años en el desarrollo y aplicación de la teoría del análisis de valores. Es autor de varios libros sobre valores, entre ellos: Value clarification as learning book (Hall, 1973), Efecto génesis (Hall, 
1986) y Values shift (Hall, 1995). Benjamin Tonna, por su parte, fue profesor de sociología en la Universidad de la Valletta (Malta) y presidió SEDOS, el Centro Internacional de Documentación en Roma. SEDOS es un foro abierto para los Institutos de vida consagrada, que se dedican a profundizar sobre la comprensión de la misión global. En él se fomenta la investigación y se difunde información a través de su boletín y página web.

Históricamente, la Compañía ha tenido en especial consideración el mundo educativo y durante todo el siglo XX ha trabajado con la firme intención de evaluar lo anticuado y adaptarse al nuevo contexto propio del siglo XXI. Todo este proceso culminó en Roma en 1993 donde el Padre General Peter-Hans Kolvenbach (Kolvenbach,1993, p. 2), vigésimo noveno Superior General de la Compañía de Jesús, en una de sus conferencias ante responsables de los colegios jesuitas manifestó que: «el fin de la educación de los jesuitas es la formación de hombres y mujeres para los demás, personas que desarrollen cuatro cualidades: conscientes, competentes, compasivas y comprometidas». Este discurso se materializó en la publicación por parte de Kolvenbach (Kolvenbach,1998, p. 21) donde, al referirse al objetivo último de la educación que se imparte en las instituciones promovidas por la Compañía de Jesús, se afirma que: "pretendemos formar líderes en el servicio y en la imitación de Cristo Jesús, hombres y mujeres competentes, conscientes y comprometidos en la compasión». Los cuatro rasgos o cualidades mencionados por Kolvenbach son fruto de la antropología educativa inspirada en la espiritualidad ignaciana, la que atribuye al perfil de estudiante de los centros educativos de la Compañía de Jesús unos principios centrados en vivir para los demás, es decir, estudiantes que no vivan para sí, sino para Dios y para su sociedad. Tal y como explica (Kolvenbach, 1998), estas cuatro metas sintetizan el sentido de la vida de la persona que pone los dones personales al servicio de los demás.

El presente artículo expone la relación existente entre el modelo HallTonna y el paradigma pedagógico ignaciano y trata de explicar cómo el modelo puede suponer una herramienta de aprendizaje clave para el desarrollo en valores individuales y organizacionales que ayuden a la transformación de los colegios jesuitas en organizaciones que aprenden. Asimismo, el artículo muestra cómo el trabajo con el modelo Hall-Tonna puede facilitar el desarrollo y crecimiento de los colaboradores de los colegios jesuitas en relación con las cuatro Cs. 


\section{EL MODELO HALL-TONNA, EL PARADIGMA PEDAGÓGICO IGNACIANO Y SU VINCULACIÓN CON LA ORGANIZACIÓN QUE APRENDE}

La organización que aprende es definida por Gairín (Gairín, 2000, p. 67) como «aquella que facilita el aprendizaje de todos sus miembros, continuamente se transforma a sí misma y resalta el valor del aprendizaje como una de las bases fundamentales de la organización». El desarrollo de la organización necesita del desarrollo de las personas y de su capacidad para incorporar nuevas formas de trabajar a la institución de la que forman parte. La organización que aprende es aquella que es capaz de reconocer y evaluar las experiencias pasadas, llevar toda esa información al presente, corrigiendo errores, resolviendo problemas, aclimatándose a las nuevas necesidades del contexto y finalmente, creando nuevas formas de proceder, innovadoras, creativas y transformadoras, que repercutan en el clima y en la cultura de la organización. En todo este proceso comentado con anterioridad, el modelo Hall-Tonna puede ser una herramienta que sirva como mapa para el crecimiento personal e institucional, desde el lenguaje de los valores.

García y Dolan (García y Dolan, 1997, p. 102) definen los valores como las «palabras que tienen un especial potencial para dar sentido y encauzar los esfuerzos humanos; a nivel personal y grupal, siendo ejes que orientan al individuo para poder emprender la acción». Maslow (Maslow, 1994, p. 21) describió los valores como una «jerarquía de necesidades» y, en este sentido, Arana y Batista (Arana y Batista, 1999, p. 4) exponen que: «cada ser humano interioriza aquello que satisface sus necesidades personales $y$, sobre esta base, posee intereses entendidos como necesidades hechas conciencia, formando convicciones que precisan y dirigen las acciones humanas de forma consciente». Para Hall (Hall, 2004, p. 7), «los valores son ideales que dan significado a la vida, son reflejados a través de las prioridades mediante las que se elige y conforme a los cuales se actúa consistente y repetidamente».

El modelo Hall-Tonna considera los valores como elementos básicos de la naturaleza humana, los cuales se representan a través de palabras y se convierten en conceptos significativos que construyen e impulsan todas las decisiones y acciones humanas. Las palabras que aluden a valores representan el interior de la persona y son imágenes o ideales que forman la base de los comportamientos externos. Las prioridades de cada persona se construyen como resultado de la visión que se tiene del mundo y del papel que se conserva en él «a medida que nos desarrollamos y crecemos las prioridades de valor cambian, generando que una nueva visión del mundo se expanda en nosotros» (Hall, 1995, p. 32). 
Una de las metas de la Compañía de Jesús es hacer énfasis en una fe que promueva la justicia. Pedro Arrupe (Arrupe,1983, p. 32), Prepósito General de la Compañía de Jesús entre 1965 y 1983, hablaba de: «formar a personas que se orientaran hacia los demás, individuos que no vivan para sí, sino para y con los demás». Por su parte, Kolvenbach (Kolvenbach, 1993, p. 61) explicaba que:

El crecimiento constante en los valores de la verdad debe conducir a una vida de plenitud y bondad, lo cual disponga a la persona a un desarrollo que se distinga por su competencia, integridad y compasión, siendo el primer paso de este desarrollo el conocimiento de uno mismo.

Los valores son ideales que significan la vida de las personas, pero ¿pueden las organizaciones y los individuos mejorar su autoconocimiento y fomentar su desarrollo de la mano de los valores? y ¿qué papel tiene el paradigma pedagógico ignaciano y el modelo Hall-Tonna en dicho proceso?

Cuando se habla sobre las organizaciones que aprenden hay que tener en cuenta una condición sine qua non: no son las organizaciones en sí mismas las que obtienen aprendizaje, sino las personas que las componen y dirigen. A pesar de lo anterior, la cultura que emana de la forma de ser y proceder de las personas en la institución sí es susceptible de ser transformada, cambiada o mejorada. La escuela, afectada por su entorno y por las personas que participan en ella, crea una cultura propia que está construida por valores, ideas establecidas, visiones y normas, según las cuales las personas se orientan para desenvolverse dentro de la misma. Esto es lo que Bolívar (Bolívar, 1996) explica que es la cultura escolar, que aparece como un factor determinante del contexto y naturaleza que acompaña a los centros educativos, que puede ayudar o dificultar el proceso de aprendizaje colectivo. Manifiesta Gairín al respecto (Gairín, 2000, p. 76):

La incidencia que tiene el entorno, la especial manera que incide en el modo en el que se relacionan y comunican sus componentes, la acción diferenciada de la dirección, los valores compartidos, la forma como se aplica el proceso organizativo, la propia historia institucional y sus inquietudes en relación con la mejora, configuran diferencias y dan una personalidad única y particular a cada institución.

Del interior de cada colegio como organización emanan valores, algunos seleccionados a propósito y otros no. Los valores se relacionan con la visión que se tiene del mundo, lo que permite crear estrategias para implementar metas que ayudan a los miembros de la organización a diseñar y a poner en marcha los sistemas de gestión para alcanzar objetivos estratégicos. Los individuos identificados con la institución donde trabajan llevan a cabo 
acciones que apoyan el logro de la visión común, y cuando esto se hace con una clara comprensión de los valores fundamentales de la organización, se posibilita el crecimiento. Sin embargo, todo lo anterior no sería posible si no existe una clara comprensión de los valores compartidos, puesto que el crecimiento personal no supondría el crecimiento de la organización y viceversa. Para evitar esta situación, es recomendable el trabajo con el modelo de análisis de valores Hall-Tonna y con el paradigma pedagógico ignaciano.

La pedagogía ignaciana en la actualidad se fundamenta en un corpus teórico reflejado en publicaciones como Características de la educación de la Compañía de Jesús (Comisión Nacional de Educación, 1986) y Pedagogía ignaciana, un planteamiento práctico (Consejo Internacional de la Educación de la Compañía de Jesús, 1996). Es en esta publicación de 1996 donde se expone el paradigma pedagógico ignaciano (PPI) con el cual se pretende situar a la persona y a la organización en el desarrollo de unos determinados valores partiendo de la experiencia, la reflexión y la acción. El modelo educativo actual de la Compañía de Jesús se organiza en cinco pasos diferenciados: $1{ }^{\circ}$ contexto, $2 .^{\circ}$ experiencia, $3 .^{\circ}$ reflexión, $4 .^{\circ}$ acción y $5 .^{\circ}$ evaluación, los cuales también tienen su representación en la morfología del modelo Hall-Tonna.

\section{TABLA 1. RELACIÓN EXISTENTE ENTRE LOS PASOS DEL PPI Y EL MODELO H-T}

\begin{tabular}{|l|l|}
\hline $\begin{array}{c}\text { Paradigma Pedagógico } \\
\text { Ignaciano }\end{array}$ & \multicolumn{1}{|c|}{ Modelo Hall-Tonna } \\
\hline $1 .^{\circ}$ Contexto & $\begin{array}{l}\text { Hace explícita la información tácita del contexto y pone } \\
\text { en relieve los valores que emanan de las conductas, } \\
\text { prioridades y situaciones concretas del sujeto o grupo. } \\
\text { Muestra la visión y valores de la persona promoviendo } \\
\text { el desarrollo, haciendo al individuo consciente de cómo } \\
\text { es y lo que busca en la realidad. }\end{array}$ \\
\hline $2 .^{\circ}$ Experiencia & $\begin{array}{l}\text { Relaciona y enfrenta la información obtenida y los } \\
\text { conocimientos sobre sí mismo del individuo en } \\
\text { lenguaje de valores, lo que produce aprendizaje. Une } \\
\text { experiencias previas a valores y las trasporta de vuelta } \\
\text { a la realidad, buscando la relación existente con las } \\
\text { conductas presentes. }\end{array}$ \\
\hline
\end{tabular}




\begin{tabular}{|l|l|}
\hline $3^{\circ}$ Reflexión & $\begin{array}{l}\text { La introspección y reflexión en la persona es uno de } \\
\text { los elementos de mayor importancia en la metodología }\end{array}$ \\
Hall-Tonna, permitiendo así discernir en valores. \\
Es en este paso donde se clarifica la motivación y \\
las prioridades de la persona para orientarse hacia \\
valores más evolucionados, obteniendo un mayor \\
conocimiento sobre cómo es, qué desea y qué pretende \\
para ella misma en la vida.
\end{tabular}

Fuente: elaboración propia.

La Compañía de Jesús promueve el desarrollo personal a través de la capacidad de interiorizar y discernir, y, a la misma vez, de experimentar la sociedad comprometiéndose con la organización donde se desarrolla. El modelo Hall-Tonna hace hincapié en la particular visión que cada persona tiene del mundo dependiendo de la fase del desarrollo donde se encuentra, vinculando dicha visión con una determinada forma de reconocerse, comprometerse y aprender. El modelo ayuda a la congruencia de valores compartidos desde las experiencias individuales, posibilitando el aprendizaje y la construcción de planes comunes de crecimiento y desarrollo en la escuela.

\section{METODOLOGÍA DEL MODELO HALL-TONNA}

Hall y Tonna identificaron y definieron 125 palabras referidas a valores, que se encuentran presentes en el lenguaje de los individuos y grupos, generando un instrumento de medición que identifica las prioridades de valores con el propósito de favorecer el desarrollo humano. Las herramientas de análisis del modelo son dos: 
- El Inventario Hall-Tonna (HTINVE), un cuestionario de 125 preguntas de aplicación individual que puede ser procesado grupalmente obteniendo un perfil de compuesto.

- Software de Análisis de Documentos (HTDOC), herramienta informática que ayuda a la identificación de valores en documentos escritos.

El modelo Hall-Tonna organiza y jerarquiza los valores de la siguiente manera (Values Technology, 2001, p. 13):

1. Tres áreas: base, central y futuro.

- Área base: valores que tienden a no dejar a la persona avanzar si no están debidamente atendidos.

- Área central: relacionada con los valores que mayor prevalencia tienen en la persona en el presente, por lo que deben obrar como instrumentos posibilitadores para alcanzar los valores de futuro.

- Área futuro: aspiraciones, aquellos valores que hacen avanzar a la persona.

Estas tres áreas se descomponen en ocho etapas que ofrecen información sobre las diferentes formas de vivir los valores:

a. Etapas personales (impares): son clave del crecimiento psicológico y espiritual y atienden al ámbito personal.

- a.1 Etapa 1: supervivencia.

- a.2 Etapa 3: familia.

- a.3 Etapa 5: vocación.

- a.4 Etapa 7: sabiduría.

b. Etapas institucionales (pares): se experimentan en un contexto grupal, son aprendizajes que se desarrollan en contacto con otras personas.

- b.1 Etapa 2: seguridad.

- b.2 Etapa 4: institución.

- b.3 Etapa 6: colaboración.

- b.4 Etapa 8: orden mundial.

Cada una de estas etapas está construida por valores medio y valores meta. Los primeros se refieren a destrezas o conductas que ayudan a la persona a evolucionar. Los segundos se relacionan con las finalidades o principios que la persona quiere conseguir. Las ocho etapas se incluyen dentro de cuatro fases del desarrollo (corresponden dos etapas por cada una de las fases), según las cuales se experimentan los valores de una determinada manera, lo que supone una particular visión del mundo. 
- Fase 1: supervivencia.

- Fase 2: pertenencia.

- Fase 3: autoiniciación.

- Fase 4: interdependencia.

TABLA 2. FASES DEL MODELO HALL-TONNA Y VISIÓN DEL MUNDO QUE CONSERVA LA PERSONA

\begin{tabular}{|c|c|}
\hline $\begin{array}{l}\text { Fases del modelo } \\
\text { Hall-Tonna }\end{array}$ & $\begin{array}{l}\text { Visión de la organización y el aprendizaje en cada } \\
\text { fase }\end{array}$ \\
\hline $\begin{array}{l}\text { 1. }{ }^{\text {a }} \text { Fase del desarrollo: } \\
\text { supervivencia }\end{array}$ & $\begin{array}{l}\text { Las personas que se encuentran en esta fase del } \\
\text { desarrollo tienen una imagen de la organización } \\
\text { amenazante de la que no se tiene gran control. El } \\
\text { individuo tiende a realizar su trabajo por el miedo a } \\
\text { la autoridad puesto que no dispone de capacidades } \\
\text { para reconducir problemas y situaciones de conflicto. } \\
\text { Se encuentra dificultad en compartir las experiencias, } \\
\text { interiorizarlas y aprender de ellas. La prioridad es uno } \\
\text { mismo. Los valores más representativos de esta fase son: } \\
\text { Interés en uno mismo, Autopreservación, Seguridad, } \\
\text { Asombro / Destino. }\end{array}$ \\
\hline $\begin{array}{l}\text { 2. }{ }^{\text {a }} \text { Fase del desarrollo: } \\
\text { pertenencia }\end{array}$ & $\begin{array}{l}\text { En esta fase, la persona percibe la organización como } \\
\text { un lugar donde existen problemas y hay que resolverlos. } \\
\text { Se relaciona el éxito a través de la pertenencia que se } \\
\text { obtiene aceptando la normativa establecida del grupo } \\
\text { por la necesidad de ser querido. El prestigio es piedra } \\
\text { angular del éxito y los valores en esta fase se centran en } \\
\text { el interés por uno mismo y por sobrevivir. La persona } \\
\text { aprende, pero no comparte sus nuevos conocimientos } \\
\text { ni trabaja de modo cooperativo. El individuo obedece } \\
\text { a la autoridad representada por las personas que } \\
\text { ocupan posiciones de poder con la finalidad de sentirse } \\
\text { reconocido. Los valores más representativos de esta fase } \\
\text { son: Familia / Pertenecer, Autoestima, Competencia / } \\
\text { Confianza, Creencia /Filosofía, Ser querido. }\end{array}$ \\
\hline
\end{tabular}




\begin{tabular}{|c|c|}
\hline $\begin{array}{l}\text { 3. a Fase del desarrollo: } \\
\text { autoiniciación }\end{array}$ & $\begin{array}{l}\text { En esta tercera fase, la organización es reconocida como } \\
\text { un lugar idóneo para desarrollar el proyecto creativo } \\
\text { en el cual la persona se siente como un individuo que } \\
\text { tiene algo único que ofrecer. La persona se encuentra } \\
\text { en un momento vital de profunda maduración que le } \\
\text { lleva a emprender dentro de la organización. No existe } \\
\text { un pensamiento de la organización como un sistema de } \\
\text { sistemas que se necesitan unos a otros, sin embargo, se } \\
\text { produce un énfasis en el crecimiento personal a través } \\
\text { de valores como: Búsqueda/Significado/Esperanza, } \\
\text { Fe/Riesgo/Visión, Discernimiento, Congruencia, } \\
\text { Construcción/Orden Nuevo, Dignidad Humana. }\end{array}$ \\
\hline $\begin{array}{l}\text { 4. }{ }^{\text {a }} \text { Fase del desarrollo: } \\
\text { interdependencia }\end{array}$ & $\begin{array}{l}\text { Esta fase presenta un contexto global en el que } \\
\text { las personas trabajan para animar y cuidar a los } \\
\text { individuos y a las comunidades. Se entiende el } \\
\text { mundo como la suma de todos que se fundamenta } \\
\text { en relaciones sistémicas en el que cada persona tiene } \\
\text { un gran valor y existe un compromiso de servir a los } \\
\text { demás. Esta visión nace de valores propios como la } \\
\text { trascendencia, la interdependencia o los derechos } \\
\text { humanos. Se adquiere el liderazgo de servicio, es } \\
\text { decir, servidor antes y por eso, líder. «Todo comienza } \\
\text { con el sentimiento natural de servir, y entonces, de esta } \\
\text { elección consciente resulta una aspiración de ayudar } \\
\text { a los demás, lo que responde a una llamada colectiva } \\
\text { para trabajar por la armonía global, construir y renovar } \\
\text { la faz de la tierra» (Hall, 1995, p. 58). Nace entonces } \\
\text { una nueva conciencia y visión del mundo basado en } \\
\text { la armonía y el equilibrio personal. Los valores más } \\
\text { representativos de esta fase son: Verdad / Sabiduría, } \\
\text { Interdependencia, Trascendencia / Soledad, Derechos } \\
\text { Humanos, Armonía Global, Visión Profética, Derechos } \\
\text { humanos, Justicia mundial. }\end{array}$ \\
\hline
\end{tabular}

Fuente: elaboración propia.

Por último, los siete ciclos están formados por dos etapas, una personal y otra institucional, contiguas en el mapa de valores. Escriben y muestran la integración de lo personal y lo institucional, una de las claves del crecimiento en el modelo Hall-Tonna.

- Ciclo 1: supervivencia-seguridad.

- Ciclo 2: seguridad-familia.

- Ciclo 3: familia-institución. 
- Ciclo 4: institución-iniciativa personal.

- Ciclo 5: iniciativa personal-nuevo orden.

- Ciclo 6: nuevo orden-sabiduría.

- Ciclo 7: sabiduría-orden mundial.

TABLA 3. ESTILOS DEL LIDERAZGO DEL MODELO HALL-TONNA Y SUS VALORES PRIORITARIOS

\begin{tabular}{|c|c|c|c|}
\hline $\begin{array}{l}\text { Estilos del } \\
\text { Liderazgo: }\end{array}$ & $\begin{array}{c}\text { Ciclos del } \\
\text { Desarrollo: }\end{array}$ & $\begin{array}{l}\text { Número } \\
\text { de Fase }\end{array}$ & Valores prioritarios \\
\hline Autocrático & $\begin{array}{l}\text { 1. }{ }^{\circ} \text { Supervivencia- } \\
\text { seguridad. }\end{array}$ & $1 .^{\mathrm{a}}$ & $\begin{array}{l}\text { Seguridad. } \\
\text { Economía/ Beneficios. } \\
\text { Autopreservación. }\end{array}$ \\
\hline Benevolente & $\begin{array}{l}\text { 2. Seguridad- } \\
\text { familia. }\end{array}$ & $1 .^{\mathrm{a}} \mathrm{y} 2 .^{\mathrm{a}}$ & $\begin{array}{l}\text { Autoestima. } \\
\text { Obediencia/deber } \\
\text { Apoyo semejantes. }\end{array}$ \\
\hline Gestor & $\begin{array}{l}\text { 3. }{ }^{\circ} \text { Familia- } \\
\text { institución. }\end{array}$ & $2 .^{a}$ & $\begin{array}{l}\text { Competencia/confianza. } \\
\text { Economía/éxito. } \\
\text { Gestión/responsable. }\end{array}$ \\
\hline Facilitador & $\begin{array}{l}4^{\circ} \text { Institución- } \\
\text { iniciativa } \\
\text { personal. }\end{array}$ & $2 .^{\mathrm{a}}$ y $3 .^{\mathrm{a}}$ & $\begin{array}{l}\text { Igualdad/liberación. } \\
\text { Servició/vocación. } \\
\text { Decisión/iniciativa. } \\
\text { Autoridad/honestidad. } \\
\text { Adaptabilidad/flexibilidad. }\end{array}$ \\
\hline Colaborador & $\begin{array}{l}5 .^{\circ} \text { Iniciativa } \\
\text { personal-nuevo } \\
\text { orden. }\end{array}$ & $3 .^{\mathrm{a}}$ & $\begin{array}{l}\text { Dignidad Humana. } \\
\text { Fe/riesgo/visión. } \\
\text { Justicia. } \\
\text { Compromiso ético. } \\
\text { Responsabilidad social. } \\
\text { Colaboración. } \\
\text { Compartir/escuchar/confiar. }\end{array}$ \\
\hline Servidor & $\begin{array}{l}\text { 6. Nuevo orden- } \\
\text { sabiduría: }\end{array}$ & $3 .^{\mathrm{a}} \mathrm{y} 4 .^{\mathrm{a}}$ & $\begin{array}{l}\text { Verdad /sabiduría. } \\
\text { Colaboración. } \\
\text { Visión profética. } \\
\text { Sinergia. } \\
\text { Trascendencia. }\end{array}$ \\
\hline Visionario & $\begin{array}{l}\text { 7. Sabiduría- } \\
\text { orden mundial. }\end{array}$ & $4 .^{\mathrm{a}}$ & $\begin{array}{l}\text { Interdependencia. } \\
\text { Palabra. } \\
\text { Macroeconomía. }\end{array}$ \\
\hline
\end{tabular}

Fuente: elaboración propia. 
Cada uno de los ciclos de desarrollo del modelo conlleva la presencia de unos valores prioritarios que desembocan en un estilo de liderazgo particular. Hall y Tonna encontraron un claro vínculo entre el liderazgo y el papel de los valores en su ejercicio, puesto que, si las prioridades de valor del líder están relacionadas con la supervivencia o la autopreservación, entonces esa persona estará motivada y predispuesta a buscar maneras de proteger su territorio y asegurar su posición. Sin embargo, si las prioridades están orientadas hacia el crecimiento y la creación de nuevas instituciones que mejoren la dignidad humana, entonces ese líder buscará maneras de incrementar las capacidades de auto proyección de quienes le rodean y adoptará estilos más colaborativos, promoviendo la creación de nuevos líderes. Al conocer sus prioridades de valor, la persona puede reconocer su estilo de liderazgo con su correspondiente ética y comportamientos primarios asociados a él.

En el modelo Hall-Tonna se explica que los valores actúan como metas a lograr en el futuro y representan la visión. A modo de ejemplo, el conocimiento juega el papel de valor meta y la investigación, en su caso, es un valor medio del cual se hace uso para alcanzar la meta. "Para que las personas actúen movidas por valores de orden superior es preciso realizar determinados itinerarios o recorridos, en los que necesariamente deben estar presentes valores de una etapa anterior, esta es la síntesis del modelo» (Elexpuru, Yániz y Villardón, 2013, p. 11).

La esencia del modelo reside en entender que, para que la persona llegue a experimentar aprendizajes de orden superior a nivel individual y grupal, los valores de mayor complejidad, más evolucionados, deben estar sustentados en valores de etapas previas que sirvan como andamiaje para desenvolverse con plenitud. Por ejemplo, si un docente tiene como objetivo implantar una metodología cooperativa en su aula necesita conservar y utilizar otros valores prioritarios como: Adaptabilidad/flexibilidad y Compartir/escuchar/ confiar. Tal y como manifiesta Maslow (Maslow, 1994, p. 52):

Para averiguar qué se debe hacer es necesario descubrir qué y cómo uno es, porque el camino hacia decisiones éticas y de valor, hacia elecciones más acertadas, hacia lo que se desea ser, pasa por lo que uno mismo ya es, por el descubrimiento de los hechos de la verdad. De trata de la fusión deliberada y voluntaria entre hechos, conductas y valores.

Para el crecimiento interior y el discernimiento, según se propone en el modelo Hall-Tonna, la persona debe disponer de una imagen clara de los valores actuales con los que se impulsa. Al entender estas prioridades de valores, el individuo obtiene un profundo conocimiento sobre sus propias necesidades y eso le ayuda a elegir, a discernir. A continuación, y a modo de resumen, se muestra un esquema de la metodología del modelo: 


\section{FIGURA 1. METODOLOGÍA DEL MODELO HALL-TONNA, VALUES TECHNOLOGY (2001)}

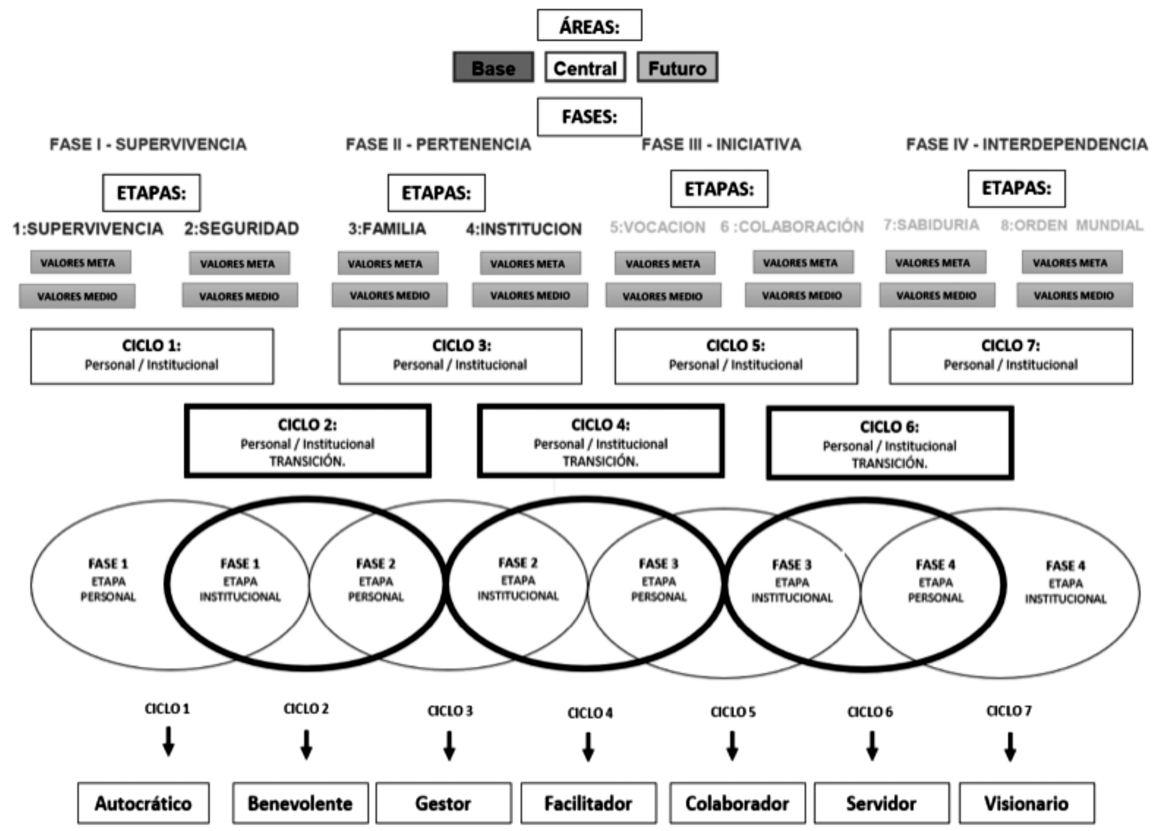

\section{LAS CUATRO CS, LOS COLEGIOS JESUITAS Y EL PAPEL DEL MODELO HALL-TONNA}

Históricamente, la pedagogía ignaciana ha combinado la excelencia con la adaptación a momentos, lugares y personas, tal y como indica Ochoa de la Fuente (Ochoa de la Fuente, 2015, p. 41):

Durante siglos, la expansión y la diversidad de los colegios jesuitas y los mecanismos de gobierno de la Compañía de Jesús aseguraban la pertenencia a una identidad común que partía del documento Ratio Studiorum (RS) 1599, en el cual se incluía un currículo organizado con una metodología pedagógica específica y una organización escolar concreta.

Del RS emanaban una serie de valores que eran trasladados a todos los jóvenes que se desarrollaban en las instituciones jesuitas, sin embargo, con el paso de los años, según señala Jesuitas Educación (Jesuites, 2014, p. 11) «el 
RS dejó de representar una referencia real que orientara los proyectos educativos de los colegios, debido quizá a la proliferación de currículos oficiales y legislaciones educativas». A pesar de haber sido reelaborado, el RS no ha conseguido mostrar la misión educativa contemporánea de los jesuitas, «quizá por la incipiente disminución del número de jesuitas en los colegios de los países desarrollados, con el consiguiente protagonismo de personas laicas comprometidas con la misión» (Ochoa de la Fuente, 2015, p. 42).

La publicación, en 1986, del libro: Características de la educación de la Compañía de Jesús (Comisión Nacional de Educación, 1986) despertó un renovado interés entre los profesionales de la educación y las familias por profundizar en los valores del estilo jesuita de enseñanza. El documento, traducido a 13 lenguas, fue el germen de diferentes seminarios y formaciones, pero, a pesar de ello, esta publicación no consiguió trascender y llevar a la práctica lo que teóricamente ponía en relieve. Años después, dentro del Congreso Pedagógico Ignaciano de 2005, del que nació el plan estratégico de 2008-2012, se ahondó en la necesidad de profundizar en el autoconocimiento y el conocimiento mutuo como herramientas para el discernimiento en valores. Posteriormente a la realización del congreso, en 2005, el Padre General Adolfo Nicolás SJ (Nicolás, 2011, p. 7) escribió un artículo titulado Retos en la educación superior jesuita hoy, en el que resaltaba que «los jesuitas estamos en educación porque, desde el principio, la gran preocupación de San Ignacio y sus compañeros y seguidores fue el crecimiento de la persona y la transformación de la persona». En 2012 se puso en marcha el Coloquio Internacional de la Educación Secundaria Jesuita (ICJSE), en el que más de cuatrocientos participantes de todo el mundo se reunieron para reflexionar sobre los retos actuales para la educación de la Compañía de Jesús. En 2014, en el Seminario Internacional sobre Pedagogía y Espiritualidad Ignaciana (SIPEI) en coordinación con la ICAJE (la Comisión Internacional por el Apostolado de Educación Jesuita), se ahondó en algunas de las tendencias pedagógicas más recientes y significativas y su relación con la pedagogía y espiritualidad ignaciana. Todo el trabajo llevado a cabo en el seminario desembocó, por ejemplo, en el programa Horizonte 2020, un revolucionario proyecto planteado por los colegios jesuitas de Cataluña que recoge algunas de las innovaciones más importantes planteadas durante los últimos años y que se centra en una educación humanista de calidad, teniendo como fin último el desarrollo de las cuatro Cs.

El actual Padre General de la Compañía de Jesús, Arturo Sosa (Sosa, 2019, p. 5), expresa en el prólogo del libro La Pedagogía Ignaciana: textos clásicos y contemporáneos sobre la educación de la Compañía de Jesús: "Un ser humano educado sabe situar sus metas personales dentro de la búsqueda del bien 
común». El modelo Hall-Tonna, por todo lo comentado con anterioridad, resulta ser una herramienta beneficiosa para ayudar a la persona a situar sus metas en la dirección de las cuatro Cs. A modo de ejemplo, en la siguiente tabla se definen las cuatro Cs y se muestran los valores, medio y meta, propios del diccionario del modelo Hall-Tonna que correlacionan con estas:

\section{TABLA 4. RELACIÓN DE LAS CUATRO CS DE LA COMPAÑÍA DE JESÚS Y EL MODELO HALL-TONNA}

\begin{tabular}{|c|c|c|}
\hline Cuatro Cs: & Definición: & $\begin{array}{l}\text { Valores en el modelo Hall- } \\
\text { Tonna que desarrollan estas } \\
\text { capacidades: }\end{array}$ \\
\hline Consciente: & $\begin{array}{l}\text { Personas que profundizan } \\
\text { en el autoconocimiento } \\
\text { y la interioridad } \\
\text { entendiendo la vida } \\
\text { como un don recibido. } \\
\text { Contemplación ignaciana } \\
\text { para descubrir a Dios, } \\
\text { llegando a la conclusión } \\
\text { de que amar y servir es } \\
\text { una respuesta por la vida } \\
\text { recibida. Compromiso } \\
\text { por cuidar y mejorar el } \\
\text { mundo desde la acción y la } \\
\text { responsabilidad. }\end{array}$ & $\begin{array}{l}\text { 1. Asombro. } \\
\text { 2. Fe/Riesgo/Visión. } \\
\text { 3. Verdad/Sabiduría. } \\
\text { 4. Libertad, Igualdad/ } \\
\text { Liberación. } \\
\text { 5. Discernimiento. } \\
\text { 6. Ética/Responsabilidad. } \\
\text { 7. Trabajo/Labor. } \\
\text { 8. Servicio/Vocación. } \\
\text { 9. Colaboración. } \\
\text { 10. Complementariedad, } \\
\text { Sinergia. } \\
\text { 11. Armonía Global. } \\
\text { 12. Palabra. }\end{array}$ \\
\hline Competente: & $\begin{array}{l}\text { Conocimiento con } \\
\text { capacidad de acción } \\
\text { exitosa, ser competente } \\
\text { significa no defraudar } \\
\text { a otros que buscan } \\
\text { los buenos servicios } \\
\text { de esa competencia. } \\
\text { Discernimiento como } \\
\text { herramienta para educar } \\
\text { personas críticas y } \\
\text { libres, para enseñar a } \\
\text { la persona a convivir, } \\
\text { a crear comunidad, } \\
\text { comprometiéndola con el } \\
\text { bien común en favor de } \\
\text { la convivencia fraternal } \\
\text { entre todas las personas. }\end{array}$ & 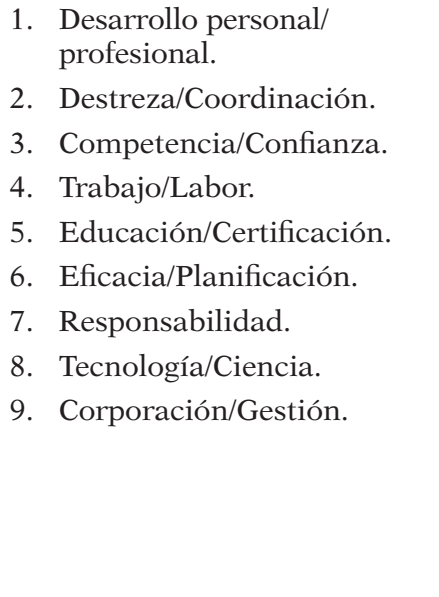 \\
\hline
\end{tabular}




\begin{tabular}{|c|c|c|}
\hline Comprometida: & $\begin{array}{l}\text { Compromiso creativo } \\
\text { que lleva a la persona a } \\
\text { responder a su medio con } \\
\text { inteligencia, voluntad } \\
\text { y afecto. Se educa para } \\
\text { servir a la sociedad en la } \\
\text { búsqueda de la justicia, } \\
\text { lo bueno y lo equitativo. } \\
\text { Ser hombre o mujer } \\
\text { para todos los tiempos, } \\
\text { lugares y personas. }\end{array}$ & $\begin{array}{l}\text { 1. Ecoridad, Armonía global. } \\
\text { 2. Justicia mundial, Derechos } \\
\text { humanos. } \\
\text { 3. Expresividad/Libertad, Ser } \\
\text { uno mismo. } \\
\text { 4. Generosidad/Compasión. } \\
\text { 5. Servicio/Vocación. } \\
\text { 6. Fe/Riesgo/Visión. } \\
\text { 7. Equidad/Derechos. }\end{array}$ \\
\hline Compasiva: & $\begin{array}{l}\text { Amar al prójimo como a } \\
\text { uno mismo significa que } \\
\text { no solo se reconoce la } \\
\text { vida propia, sino también } \\
\text { se ama la de los otros. } \\
\text { La persona es cuidadora } \\
\text { y responsable de los } \\
\text { demás, por lo que es } \\
\text { necesaria la sensibilidad } \\
\text { para ver y responder a } \\
\text { las necesidades del otro. } \\
\text { Reconocer, afirmar y } \\
\text { amar la vida y dignidad } \\
\text { del otro se convierte en el } \\
\text { sentido central de la vida } \\
\text { propia. }\end{array}$ & $\begin{array}{ll}\text { 1. } & \text { Empatía. } \\
\text { 2. } & \text { Equidad/Derechos. } \\
\text { 3. } & \text { Generosidad/Compasión. } \\
\text { 4. } & \text { Búsqueda/Significado/ } \\
& \text { Esperanza. } \\
\text { 5. } & \text { Contemplación. } \\
\text { 6. } & \text { Colaboración. } \\
\text { 7. } & \text { Responsabilidad/ } \\
\text { Compartida. } \\
\text { 8. Verdad/Sabiduría. } \\
\text { 9. Interdependencia. } \\
\text { 10. Visión profética. } \\
\text { 11. Sinergia. } \\
\text { 12. Derechos humanos. } \\
\text { 13. Justicia mundial. }\end{array}$ \\
\hline
\end{tabular}

Fuente: elaboración propia, a partir de la publicación de Ugalde (2016).

\section{CONCLUSIONES}

1. El modelo Hall-Tonna tiene como principal misión promover el desarrollo humano ayudando a la persona a crecer a través del discernimiento en valores. Los valores propios de las etapas más evolucionadas del modelo coinciden con la esencia y las características de cada una de las cuatro cualidades jesuitas. Aquellas personas que se sitúan en etapas evolucionadas (seis, siete y ocho) del modelo, centradas en la fase IV, tienen una visión del mundo en forma de contexto global en el que las personas trabajan para animar y cuidar a los individuos y a las comunidades, sienten una llamada a la colaboración, a trabajar por la 
armonía global, construir y renovar la faz de la tierra. En la fase IV se dan valores propios como la Trascendencia, el Servicio y los Derechos Humanos, valores intrínsecos en el mensaje de Jesús de Nazaret e Ignacio de Loyola, que están presentes en el modelo Hall-Tonna y son la base de las cuatro cualidades jesuitas.

2. Para aquellas personas que no tienen la visión del mundo que les permita el desarrollo de las cuatro Cs, el trabajo con el modelo Hall-Tonna podrá ayudarles a experimentar valores previos a esta fase, como el valor Colaboración, Desarrollo personal/profesional o Servicio/vocación. Para que la persona actúe en favor de los demás, es necesario un desarrollo personal previo, es decir, cuando la persona tiene suficientes conocimientos sobre ella misma, cuando ha experimentado valores de etapa dos: Seguridad, etapa tres: Familia / Pertenecer, etapa cuatro: Competencia / Confianza o Igualdad / Liberación, podrá ir tomando decisiones de forma autónoma, siendo más consciente de la realidad que le rodea y del papel que puede adquirir para ser líder en el servicio a los demás.

3. Una vez que los individuos aclaran sus prioridades de valor personal y sus visiones personales, pueden interactuar abiertamente en un proceso de definir valores compartidos con el grupo o institución, para después comprometerse verdaderamente con ella. De este modo, el crecimiento será mutuo. El desarrollo de la institución, en este caso un colegio, hará crecer a la persona y viceversa, dado que podrán trabajar para compartir la misma visión y parte de sus valores meta y medios.

4. El modelo puede ayudar a construir equipos efectivos que partan de una comprensión mutua. Cuando los miembros individuales del equipo completan un inventario de valores, se puede desarrollar una imagen compuesta de los valores del equipo. Los valores compuestos, agrupados en grupos de valor pasado, presente y futuro, ayudan a generar debate y entendimiento sobre los temas más importantes de su sistema u organización. El análisis de valores sirve como andamiaje para la creación de equipos más efectivos.

5. Si el liderazgo está determinado por una serie de valores prioritarios que desembocan en un estilo de liderazgo particular, tal y como se entiende en el modelo Hall-Tonna, la comprensión de dichos valores, reconociendo las fortalezas y debilidades que de ellos se desprenden, promoverá en la persona el discernimiento, creciendo en la línea propuesta con las cuatro Cs, ayudándola a innovar, adaptarse a un mundo cambiante, fortalecerse y fortalecer a los demás. 


\section{REFERENCIAS}

Arana, M., y Batista, N. (1999). La educación en valores: una propuesta pedagógica para la formación profesional. Pedagogía Universitaria, 4(3). 7-19.

Arrupe, P., y Torelló, J. M. (1983). Hombres para los demás. Barcelona: Diáfora.

Bolívar, A. (1996). Cultura escolar y cambio curricular. Bordón, 48(2), 169-177.

Bunes, M., Calzón, J., Elexpuru, I., Fañanas, T., Muñoz-Repiso, M., y Valle, J. (1993). Los valores en la LOGSE Un análisis de documentos a través de la metodología de Hall-Tonna. Bilbao: Mensajero-ICE de la Universidad de Deusto.

Consejo Internacional de la Educación de la Compañía de Jesús (1996). Pedagogía ignaciana: un planteamiento práctico. Guadalajara: ITESO.

Elexpuru, I., y Bunes, M. (1994). Los valores de la comunidad educativa y el proyecto educativo de centro. Aula de innovación educativa, 2(32), 5-10.

- (2001). Desarrollo de los valores en las instituciones educativas. Bilbao: Ministerio de Educación, cultura y deporte/ICE Universidad.

Elexpuru, I., Villardón, L., y Yániz, C. (2013). Identificación y desarrollo de valores en estudiantes universitarios. Revista de Educación, 362(2), 101-162.

Elexpuru, I., Yániz, C. y Villardón, L. (2008). Desarrollo del liderazgo de servicio a través de los valores. Innovación y cambio en las organizaciones educativas. 23(1), 515-526. País Vasco: Universidad de Deusto.

Fonseca, J. (2003). El desarrollo axiológico del profesorado y la mejora institucional. Tarragona: Universitat Rovira i Virgili.

Gairín, J. (2000) «Cambio de cultura y organizaciones que aprenden», en A. Villa (coord.), «Liderazgo y organizaciones que aprenden». Bilbao: ICE-Universidad de Deusto.

Gairín, J. (2000). La Organización Escolar: contexto y texto de actuación. Madrid: La Muralla.

García, S. y Dolan, S. (1997). Dirección por Valores. España: Mc Graw Hill.

Hall, B.P. (1973). Value clarification as learning process; a guidebook. New York: Paulist Press.

- (1986). The Genesis Effect: Personal and Organizational Transformations. New York: Paulist Press.

- (2004). The Omega Factor: A Values-Based Approach for Developing Servant Leadership. New York: Twin Lights Publishing.

Hall, B. P. (1995). Values Shift. A guide to personal and organizational transformation. New York: Twin Lights Publishing.

Jesuites. (1986). Características de la educación de la Compañía de Jesús. Argentina: Comisión Nacional de Educación.

- (1996). Pedagogía ignaciana: un planteamiento práctico. Alemania: Consejo Internacional de la Educación de la Compañía de Jesús.

- (2014), Transformando la Educación. Barcelona: Edición digital.

Kohlberg, L. (1986). Lawrence Kohlberg, consensus and controversy. Londres: Routledge.

Kolvenbach, P. H. (1993). La Pedagogía ignaciana hoy. Discurso a los participantes del grupo de trabajo sobre la Pedagogía Ignaciana, un planteamiento práctico. Villa 
Cavalletti. Recuperado de: http://cristoreyvalladolid. files. wordpress. com/2010/09/ texto-pedagogiaignaciana. pdf.

Kolvenbach, P. H. (1998). El compromiso de la Compañía de Jesús en el Sector de la Educación. Chicago: Loyola eCommons.

Maslow, A. (1994). La personalidad creadora. Barcelona: Editorial Kairós.

Nicolás, A. (2011). Challenges to Jesuit higher education today. Conversations on Jesuit Higher Education, 40(1), 5-12.

Ochoa de la Fuente, L. (2015). Creer, poder y hacer. Horizonte 2020 en las escuelas jesuitas de Barcelona. Propuesta Educativa, 2(44), 38-53.

Sosa, A. (2019). Prólogo. En S.J. Mesa (eds.), La Pedagogía Ignaciana: textos clásicos y contemporáneos sobre la educación de la Compañía de Jesús (pp.5-8). España: Mensajero, Sal terrae, Universidad Pontificia Comillas.

Ugalde, L. (2016). Educación y trabajo productivo. Anales Venezolanos de Nutrición, 7(27), 6-12.

Values Technology (2001). Mentoring Accreditation Workshop Manual. Santa Cruz: Values Technology Ltd. 